\title{
Momentum- and space-resolved high-resolution electron energy loss spectroscopy of individual single-wall carbon nanotubes
}

\author{
F. S. Hage, ${ }^{1, *}$ T. P. Hardcastle, ${ }^{1,2}$ A. J. Scott, ${ }^{2}$ R. Brydson, ${ }^{2}$ and Q. M. Ramasse ${ }^{1, \dagger}$ \\ ${ }^{1}$ SuperSTEM Laboratory, SciTech Daresbury Campus, Daresbury WA4 4AD, United Kingdom \\ ${ }^{2}$ School of Chemical and Process Engineering, University of Leeds, Leeds LS2 9JT, United Kingdom \\ (Received 26 January 2017; revised manuscript received 30 March 2017; published 11 May 2017)
}

\begin{abstract}
The ability to probe the electronic structure of individual nano-objects at high energy resolution using momentum- and space-resolved electron energy loss spectroscopy in the scanning transmission electron microscope is demonstrated through the observation of confinement of the $\pi$ plasmon in individual single-wall carbon nanotubes. While confinement perpendicular to the tube axis was identified for all investigated tubes, a variable degree of confinement parallel to the tube axis was attributed to the concentration of topological defects. Spatially resolved valence loss spectra allowed for the identification of a loss peak attributed to a chirality-dependent radial interband transition. Furthermore, the importance of a careful consideration of loss peak momentum dispersions for the interpretation of spatially resolved valence loss spectra is discussed.
\end{abstract}

DOI: 10.1103/PhysRevB.95.195411

\section{INTRODUCTION}

Since 1991, the interest in carbon nanotubes (CNTs) [1] has remained high in the scientific community. This is in part due to the wide range of electronic properties (from semiconducting to metallic) that single-wall (SW) CNTs can exhibit [2]. The electronic properties of a SWCNT are to a large degree determined by its chirality, which is commonly described by a set of chiral indices $(n, m)$ [3]. A SWCNT of a given $(n, m)$ set of indices will exhibit sharp nonsmooth peaks in the quasi-1D valence and conduction band density of states (DOS), referred to as Van Hove singularities (VHSs). Scanning tunneling spectroscopy (STS) allows for direct probing of the DOS of individual SWCNTs suspended in vacuum; however, STS measurements are reportedly afflicted by substrate effects [4]. Transitions between the sharp VHS peaks in the valence and conduction band have been observed using a range of techniques including electron energy loss spectroscopy (EELS) using a purpose-built stand-alone spectrometer apparatus [5-8], Rayleigh scattering spectroscopy (RSS) $[9,10]$, fluorescence excitation spectroscopy [11], optical absorption spectroscopy [12], and spatial modulation (optical) spectroscopy (SMS) $[13,14]$. While most of these methods lack the spatial resolution to investigate individual CNTs, measurements have been reported for individual suspended SWCNTs using STS [4], RSS [10], and SMS [13].

Due to recent advancements in transmission electron microscope (TEM) electron source monochromation [15-17], an EEL spectrometer coupled to a TEM column now allows for detailed investigations of $\pi \rightarrow \pi^{*}$ transitions between the SWCNT conduction and valence VHSs $[18,19]$. Moreover, TEM and scanning (S) TEM allow for the determination of the chiral indices of each investigated tube using either a Fourier transform (FFT) of a high-resolution image [19,20] or an electron diffraction pattern $[18,21,22]$. Note that the CNT valence loss spectrum $(\mathrm{EEL}<50 \mathrm{eV})$ does not only contain peaks corresponding to chirality-dependent $\pi \rightarrow \pi^{*}$

\footnotetext{
*fshage@ superstem.org

†qmramasse@superstem.org
}

transitions but also provides information about higher energy interband transitions involving $\sigma$ states, as well as two collective modes of the system: the $\pi$ and $\pi+\sigma$ plasmons. The dispersion of both interband transitions and collective modes across the Brillouin zone can be investigated by resolving valence loss spectra in momentum space. Indeed, SWCNT "bulk" or ensemble measurements (i.e., averaged over samples containing a large number of SWCNTs to measure) using a purpose-built stand-alone spectrometer apparatus have shown that the collective $\pi$ valence electron response comprises a nondispersive $\left(\pi_{1}\right)$ and dispersive $\left(\pi_{2}\right)$ mode [6-8]. The $\pi_{1}$ plasmon exhibits a vanishing dispersion which has been attributed to plasmon confinement perpendicular to the CNT axis [5-8], while the distinct dispersion of the $\pi_{2}$ plasmon has been interpreted in terms of a plasmon propagating along the CNT axis [6-8]. This can be understood from the freedom to vary the wave vectors continuously along the unconfined length of the tube which allows for modes of varying momentum, with no such freedom appearing for modes perpendicular to the CNT axis, where allowed wave vectors are restricted to a limited number of chirality-dependent values within a discrete set (see, e.g., [23]). Momentum-resolved EELS of related carbon materials has been reported using TEM (graphene) [24-26] and STEM (individual multilayer graphene nanocones) [27].

The present work is focused on taking advantage of the combined spatial and energy resolution of a state-of-theart monochromated STEM-EELS system to investigate the valence loss response of individual SWCNTs in real and momentum space. While momentum-resolved STEM-EELS of the carbon $K$ ionization edge has been used to map defects in individual multiwalled CNTs [28], this work is a report on plasmon dispersions acquired from individual SWCNTs using EELS. In contrast to methods where the spectroscopic signal is acquired from SWCNT "bulk samples" [5-8], STEM-EELS allows uniquely for the characterization of the electronic structure and plasmon dispersions as a function of both the chirality of individual SWCNTs and the possible presence of (atomic-sized) defects in the tubes. The present results confirm that $\pi$ plasmon confinement perpendicular to the CNT axis occurs for individual tubes. Moreover, an observed variable $\pi$ plasmon confinement parallel to the tube axis is 
attributed to the concentration of topological defects present in the individual SWCNTs probed here. Specifically, these topological defects are nonhexagonal rings incorporated in the SWCNT walls. Intriguingly, the topological-defect-induced collective mode confinement appeared to predominantly affect the $\pi$ valence electrons. This can be rationalized in terms of nonhexagonal ring defects disrupting the delocalized $\pi$ states along the tube axis, which in turn affects the collective response of the $\pi$ valence electrons. As $\sigma$ states are significantly more localized than $\pi$ states, the lack of an observed confinement of the collective response of the $\sigma$ valence electrons may be explained by $\sigma$ states being much less susceptible to isolated atomic scale defects than $\pi$ states. While the exact mechanism through which topologically induced $\pi$ plasmon confinement occurs has yet to be determined, it is suggested that the role of topological defects in CNTs, and by extension graphene, should be taken into consideration in the development of any novel CNT- or graphene-based plasmonic and optoelectronic devices. Moreover, it is proposed that topological defect engineering could be used to form plasmonic conduits and possibly more complex functional geometries in graphene. The present results highlight the advantage of combining space- and momentum-resolved valence EELS measurements. Recorded loss peak dispersions across the Brillouin zone were in particular crucial in developing a coherent interpretation of $\pi+\sigma$ plasmon peak fine structure as a function of distance to the nanotube axis, as well as for the assignment of a loss peak for a $(13,7)$ metallic SWCNT as a radial chirality-dependent interband transition.

\section{EXPERIMENTAL DETAILS}

STEM-EELS experiments were carried out using a Nion UltraSTEM100MC monochromated dedicated STEM, equipped with a Gatan Enfinium ERS spectrometer optimized for high stability. The microscope was operated at an acceleration voltage of $60 \mathrm{kV}$. The spatially resolved valence loss spectra in Figs. 2, 3, and 8 were acquired with a convergence semiangle $(\alpha)$ of $31 \mathrm{mrad}(1.0 \AA$ probe size $)$ and a spectrometer collection semiangle $(\beta)$ of 44 mrad. C- $K$ core loss spectra in Fig. 2 were acquired with $\alpha=31 \mathrm{mrad}$, $\beta=60 \mathrm{mrad}$. Momentum-resolved spectra shown in Figs. 5 and 6 were acquired using a "nanodiffraction" mode, i.e., a small, mostly parallel probe with residual convergence of $\alpha=0.9 \mathrm{mrad}$, yielding an estimated diffraction-limited probe size of $\sim 3 \mathrm{~nm}$. In Fig. 1, the medium-angle annular dark field (MAADF) images were acquired with $\alpha=31 \mathrm{mrad}$, while the diffraction patterns were acquired using the "nanodiffraction" mode $(\alpha=0.9 \mathrm{mrad})$.

The momentum selectivity of the valence loss spectra was achieved by modifying the postspecimen lens excitations as well as limiting the spectrometer acceptance angle with a rectangular spectrometer entrance slit (see, e.g., Refs. [24,25]). The slit was oriented in such a way that the narrow part of the slit was parallel to the energy-dispersive direction of the spectrometer. This results in a two-dimensional spectrum forming on the spectrometer camera, where the wave vector and EEL axes are perpendicular. This means all momentum-resolved spectra were acquired in parallel, instead of serially (see, e.g., Refs. [27,29]). Individual spectra were extracted from the full

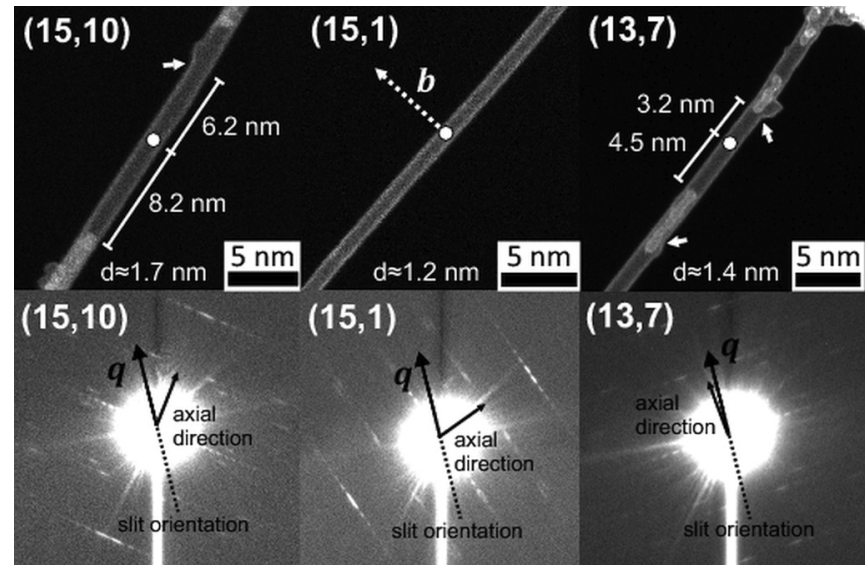

FIG. 1. MAADF images and diffraction patterns of three SWCNTs. Topological defects in the $(15,10)$ and $(13,7)$ tubes are indicated by white arrows. The momentum-selecting slit orientations and the tube axis directions are indicated in the diffraction patterns. The orientation of the tubes in the MAADF images is not directly related to the orientations of the diffraction patterns. The white disks superimposed on the MAADF images indicate the beam positions at which the spectra in Fig. 3 were acquired. The white lines superimposed on the MAADF images indicate the distance between the beam positions (white disks) and tube defects. The dashed white arrow in the $(15,1)$ tube MAADF image illustrates the experimental setup used to acquire the spectra shown in Fig. 8.

data set along the "wave vector axis," at an increment of the effective pixel size $\Delta q_{q}=0.06 \AA^{-1}$. From adding the angular spread of the electron beam $2 \alpha=0.22 \AA^{-1}$ and the effective slit width $\Delta q_{\mathrm{EEL}}=0.48 \AA^{-1}$ in quadrature, the momentum resolution was estimated to be $\Delta q \approx \pm 0.25 \AA^{-1}$.

The energy resolution, measured as the full width at half maximum of the quasielastic zero loss peak (ZLP), was $60 \mathrm{meV}$ for the spatially resolved measurements and 80-120 meV for the momentum-resolved measurements, at a dispersion on the spectrometer camera of $20 \mathrm{meV}$ per channel where $\alpha=31 \mathrm{mrad}$ and $\alpha=0.9 \mathrm{mrad}$, respectively. This difference in energy resolution is attributed mostly to an increase in uncorrected chromatic spectrometer aberrations accompanying the change in postspecimen lens setup going from the "spatially resolved" to the "momentum-resolved" beam geometry. $\mathrm{C}-K$ ionization edges in Fig. 2 were averaged across the tube diameters and acquired with an effective energy resolution of $150 \mathrm{meV}$, at a dispersion of $50 \mathrm{meV}$ per channel. The C- $K$ edges were calibrated to a nominal onset of the $\pi^{*}$ peak at $284 \mathrm{eV}$. Peak values in Tables II and III and in the plots in Fig. 7 were determined by Gaussian fitting of background-subtracted spectra. The uncertainties of the measured energy loss values in Fig. 7 are primarily attributed to choice of fitting function and fitting parameters, the magnitude of which is expected to increase with the degree of peak overlap and increasing spectral noise (in practice with increasing $\boldsymbol{q}$ ). Precisely determining these errors is problematic in the present case; however an estimate was achieved by measuring peak values from the extremes of acceptable fits for a select number of representative spectra over the entire measured momentum 


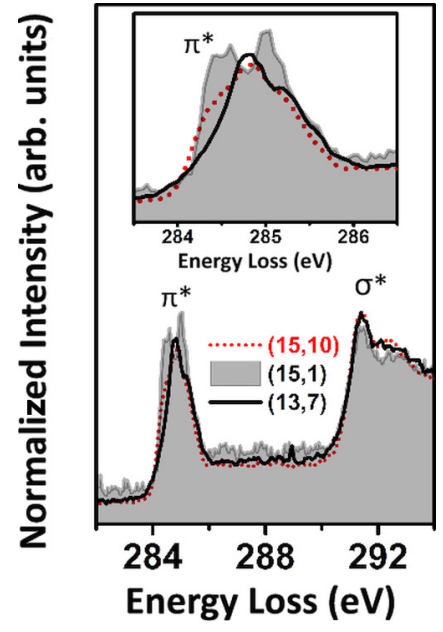

FIG. 2. Core loss C- $K$ edges from the SWCNTs.

range, for all three tubes. The resulting nonstandard errors range from $< \pm 0.02 \mathrm{eV}$ at low $\boldsymbol{q}$ to a maximum of $\sim \pm 0.2 \mathrm{eV}$ for the highest $\boldsymbol{q}$ measurements.

In Figs. 2-6 and 8, the spectral background was subtracted using a power law, except for the $(13,7)$ tube in Figs. 5 and 6 where a first-order polynomial model was used. The first-order polynomial model was likely required for adequate background subtraction in order to compensate for unresolved $\pi \rightarrow \pi^{*}$ contributions to the ZLP tail of the metallic tube spectra. Commercially available powders of SWCNTs (produced through laser ablation) were dispersed onto standard lacy carbon TEM support films after sonication in ethanol. The grid was heated to $130{ }^{\circ} \mathrm{C}$ in vacuum (pressure below $5 \times 10^{-5}$ Torr) prior to insertion into the microscope vacuum in order to prevent contamination buildup (extraneous hydrocarbons) which would otherwise risk covering the CNTs.

\section{RESULTS AND DISCUSSION}

\section{A. SWCNT chirality and defects}

Figure 1 shows diffraction patterns and MAADF STEM images of three tubes whose chiral indices were assigned to

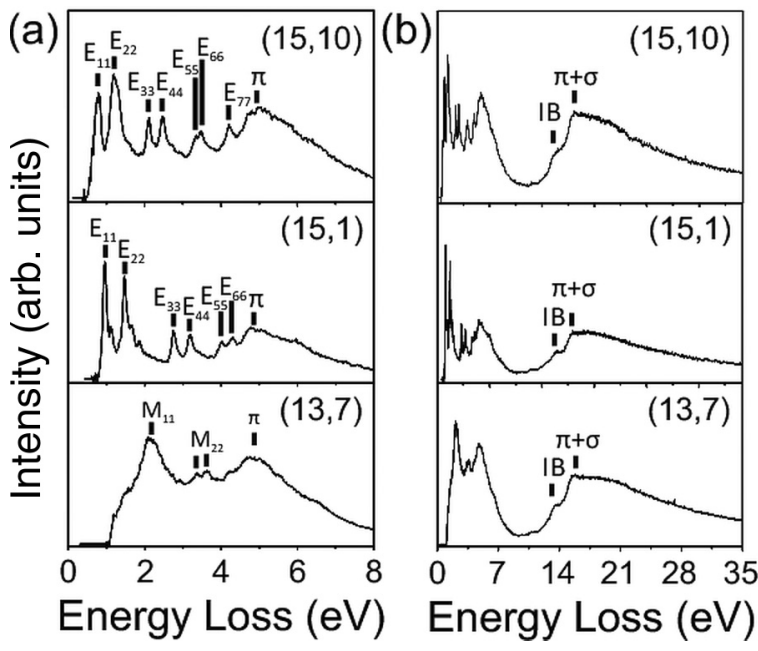

FIG. 3. Valence loss spectra from the three tubes.

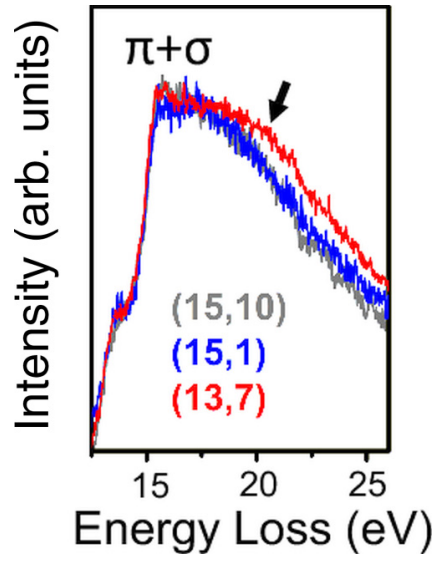

FIG. 4. Comparison of the $\pi+\sigma$ plasmon peaks from the three tubes. The black arrow indicates the spectral contribution of disordered carbonaceous material deposited on or inside the $(13,7)$ tube.

be $(15,10),(15,1)$, and $(13,7)$, as discussed below. Note that the orientation of the tubes in the MAADF images is not directly related to the orientations of the diffraction patterns. A SWCNT can be classified as either semiconducting or metallic based on its chiral index $(n, m)$ : a tube is metallic if $2 n+m=3 N$ (where $N$ is an integer); otherwise the tube is semiconducting [30]. Thus the $(15,10)$ and $(15,1)$ tubes are semiconducting while the $(13,7)$ tube is metallic. MAADF images in Fig. 1 clearly show that the tubes are single-walled and, in the case of the $(15,1)$ tube, clean and defect free. The $(15,10)$ and $(13,7)$ tubes are both sparsely covered by or containing thin layers of disordered carbonaceous material, appearing as brighter contrast regions in the MAADF images (Fig. 1). While likely primarily carbon-based, this disordered material could possibly also contain smaller amounts of other elements originating from the CNT production process. A detailed study of the carbonaceous material elemental composition was however beyond the scope of the present work. Moreover, the white arrows in the images in Fig. 1 indicate the presence of topological defects in both the $(15,10)$ and $(13,7)$ tubes. Specifically, Fig. 1 indicates that the topological defects are primarily nonhexagonal rings (see white arrows, Fig. 1) incorporated in the graphene sheets making up the walls of the CNTs. Contributions of tube imperfections to the recorded loss spectra will be discussed below.

Table I shows the chiral angles, ratio of chiral indices $(m / n)$, and individual chiral indices determined from the SWCNT diffraction patterns in Fig. 1, following Refs. [21,22]. Ratios between the position of the first and second diffraction peak of the so-called principal layer lines in a SWCNT diffraction pattern, denoted $X_{2} / X_{1}$, can be used to assign chiral indices directly [22]. The precision of all values extracted from the diffraction patterns was however limited by a combination of a finite electron beam convergence and camera dynamic range. Due to these uncertainties and sources of noise, additional information was therefore used to confirm the chiral assignment.

The carbon $K$ edge $\pi^{*}$ peak fine structure is dominated by transitions from the $1 s$ ground state to VHSs in the 

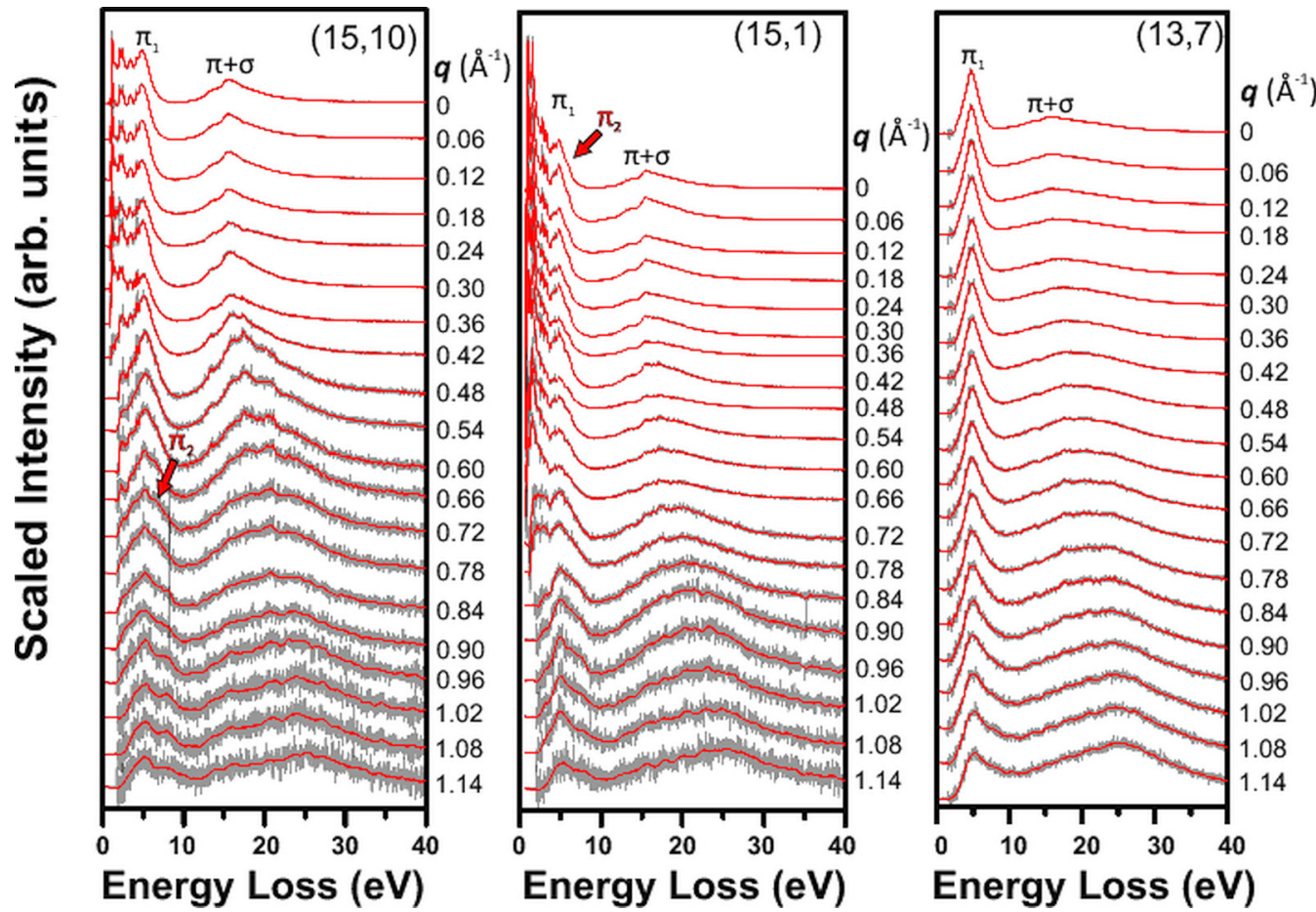

FIG. 5. ZLP-subtracted momentum-resolved valence loss spectra of the SWCNTs. Smoothed data (red) superimposed on the raw data (gray) as a guide to the eye. Spectral intensities are scaled for ease of comparison. The direction of $\boldsymbol{q}$ with respect to the CNT axial direction for each tube is indicated in the diffraction patterns in Fig. 1.

unoccupied DOS above the Fermi level, and thus is highly sensitive to tube chirality $[19,31]$. This has been demonstrated experimentally for individual SWCNTs using (S)TEM-EELS by Rossouw et al. [31] and Senga et al. [19]. Rossouw et al. [31] showed that the $\pi^{*}$ peak exhibits significantly different line shapes depending on whether an individual tube is metallic and semiconducting. Senga et al. [19] investigated several individual metallic and semiconducting tubes, demonstrating that the $\pi^{*}$ peak fine structure is significantly affected by tube chirality. Upon detailed analysis, they found excellent agreement between experimental $\pi^{*}$ peak fine structure and tight-binding calculations [19]. Thus, it is clear that the C- $K$

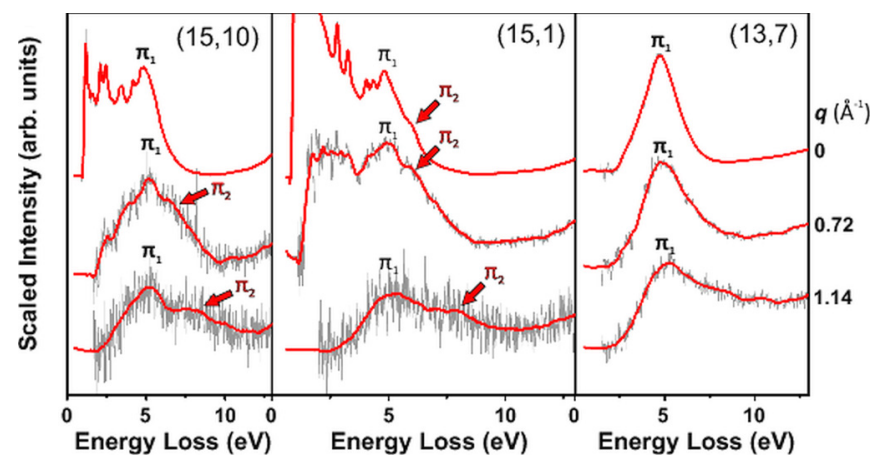

FIG. 6. Selected momentum-resolved valence loss spectra of the SWCNTs. Smoothed data (red) superimposed on the raw data (gray) as a guide to the eye. Spectral intensities are scaled for ease of comparison. The direction of $\boldsymbol{q}$ with respect to the CNT axial direction for each tube is indicated in the diffraction patterns in Fig. 1. $\pi^{*}$ peak fine structure can allow for detailed analysis of the chirality-dependent unoccupied DOS of individual SWCNTs. In the present case, Fig. 2 shows core loss spectra from the $(15,10),(15,1)$, and $(13,7)$ tubes. The $\pi^{*}$ fine structure in Fig. 2 clearly varies significantly with tube chirality. Upon comparison to the literature, the $(13,7)$ tube $\pi^{*}$ fine structure appears similar to that observed for metallic tubes in Refs. [19,31], while the $(15,1)$ and $(15,10)$ tube $\pi^{*}$ fine structures show similarities to semiconducting tubes in Ref. [19] and Refs. [19,31], respectively. A more detailed analysis of the spectra in Fig. 2 was beyond the scope of the present work but in a more general perspective, SWCNT $\mathrm{C}-K \pi^{*}$ peak fine structure analysis with a view to determine chirality directly could in some cases be complicated by factors such as limited experimental spectral resolution, lifetime broadening [19,32], and relatively small energy separations between conduction band VHSs for some tubes. Nevertheless, in comparison to valence loss spectra (discussed below), the recorded C- $K$ edge signal is highly localized [19,32,33], so that no effect from topological defects or superimposed disordered material $\geqslant 1 \mathrm{~nm}$ away is expected to influence the EEL spectrum, which presents an advantage over valence loss based assignment techniques for the analysis of local SWCNT electronic structure.

Figure 3 shows valence loss spectra acquired from each tube with the electron beam incident on the center of the tube, indicated by the white disks in Fig. 1. Tables II and III compare $\pi \rightarrow \pi^{*}$ VHS peak positions for the spectra in Fig. 3 to literature values obtained from Rayleigh scattering spectroscopy $[9,10]$, fluorescence excitation spectroscopy [11], interpolation from experimental data [9], optical absorption 
TABLE I. Experimental SWCNT structural parameters determined from the diffraction patterns in Fig. 1 (exp.) compared to the predicted values [22].

\begin{tabular}{lcccc}
\hline \hline Nanotube & Chiral angle (deg) & $m / n$ & $X_{2} / X_{1}$ & $m\left(X_{2} / X_{1}\right)[22]$ \\
\hline$(15,10)$ exp. (Ref. [22]) & $24(23.413)$ & $0.7(0.667)$ & 1.41 & $10(1.398)$ or 9 (1.428) \\
$(15,1)$ exp. (Ref. [22]) & $2(3.192)$ & $0.04(0.0667)$ & 2.89 & $1(2.892)$ \\
$(13,7)$ exp. (Ref. [22]) & $21(20.174)$ & $0.6(0.5385)$ & 1.48 & $7(1.507)$ or 8 (1.465) \\
\hline \hline
\end{tabular}

spectroscopy [12], and a prediction based on fluorescence data [34]. In agreement with Sato and Terauchi [18] the $\pi \rightarrow \pi^{*}$ VHS EELS peaks appear at up to $\approx 0.2 \mathrm{eV}$ higher energy than those measured by optical techniques. While the EEL spectrum is proportional to the loss function, the optical absorption spectrum is given by the imaginary part of the dielectric function (see, e.g., Ref. [35]). Thus the observed VHS EELS peak values can be rationalized by the contribution of the real part of the dielectric function to the loss function [32,36], effectively shifting VHS EELS peaks away from the corresponding optical values (given by maxima in the imaginary part of the dielectric function).

The nomenclature for assigning $\pi \rightarrow \pi^{*}$ transitions between VHSs in the valence loss spectrum follows that of Refs. [18,19,37]. While, to the knowledge of the authors, experimental literature values confirming the energies of the $(15,10) E_{55}-E_{77}$ and $(15,1) E_{33}-E_{66}$ peaks are lacking, these peaks were tentatively assigned to transitions between higher energy VHSs, as this is their most likely origin. Alternatively, assignment as "VHS peaks" might be done on the basis of theoretical modeling [18]; however this was beyond the scope of the present study.

Due to the so-called trigonal warping effect [23] there is a splitting of the equivalent $M_{11}$ and $M_{22}$ VHS peaks typically observed in metallic CNTs. The splitting is reflected in nomenclature where $M_{11}^{-}$and $M_{11}^{+}$(resp. $M_{22}^{-}$and $M_{22}^{+}$) correspond to the lower $(-)$ and higher energy $(+)$ VHSs EEL peaks. The magnitude of VHS peak splitting is chirality dependent [23]. Here, a clear splitting of the $M_{22}$ peak can be observed, whereas the splitting is not as pronounced for the $M_{11}$ peak, even though for $(13,7)$ SWCNTs, a $0.13-$ $0.15 \mathrm{eV}$ splitting of the $M_{11}$ peak has been reported by optical measurements $[9,12]$. The lack of an obvious splitting of the $M_{11}$ peak in the $(13,7)$ tube EEL spectrum in Fig. 3(a) is attributed to experimental factors: predominantly spectral noise and obfuscation by the intense tail of the ZLP. Overall, by comparing experimentally measured structural parameters and valence VHS peak energies to reported values [9-12,22,34] as summarized in Tables I-III, as well as comparison of the C- $K$ core-loss fine structures to the literature [19,31], it was possible to unambiguously confirm the chirality assignment of the three SWCNTs in Fig. 1.

\section{B. Valence loss EELS features in SWCNTs}

In addition to "VHS" $\pi \rightarrow \pi^{*}$ peaks, the loss spectra in Fig. 3 exhibit further features at $\approx 4.9-5.0 \mathrm{eV}, \approx 13.7-9 \mathrm{eV}$, and $\approx 15.6-15.8 \mathrm{eV}$, which are attributed to the $\pi$ plasmon [5-7,38], a sum over interband transitions (IBs) [6,7] and the $\pi+\sigma$ plasmon, respectively [5-7,38]. Plasmon peaks can be understood as arising from the incident electron beam setting up a collective oscillation including only the $\pi$ or all $(\pi+\sigma)$ valence electrons [39]. In a first approximation the "IB peak" might be attributed to a sum over $\sigma \rightarrow \sigma^{*}$ transitions $[35,40,41]$. This assignment assumes that the recorded spectrum is dominated by the $\boldsymbol{q} \rightarrow 0$ tangential response. However, due to tube wall curvature and the use of a finite spectrometer collection aperture, additional contributions to the IB loss peak from $\sigma \rightarrow \pi^{*}$ and $\pi \rightarrow \sigma^{*}$ transitions are expected. While diameter-dependent [40] and possibly other chirality-induced effects might be expected for EEL $>10 \mathrm{eV}$, qualitatively the IB peaks appear highly similar in all three spectra in Fig. 3. This might in part be due to the more intense $\pi+\sigma$ peak obscuring any IB peak fine structure.

Due to so-called inelastic delocalization [32], valence loss spectra record information from inelastic energy losses occurring up to several $\mathrm{nm}$ away from the position of the electron probe. A varying proximity of the electron beam (whose placement is indicated by the white disks in Fig. 1) to tube imperfections, such as topological defects and covering disordered carbonaceous material, allows for a comparison of the contribution of these imperfections to the spectra in Fig. 3. Any such effect will take the form of contributing spectral features characteristic of disordered carbon [42] and topological defects [43-45], that increases in relative intensity with increasing proximity to the electron beam position [32], which if present, must be taken into account in any detailed analysis. However, when comparing the spectra of the

TABLE II. Measured $\pi \rightarrow \pi^{*}$ "VHS peak" values for the $(15,10)$ and $(15,1)$ tubes, compared to reported values from Rayleigh scattering spectroscopy [9,10], fluorescence excitation spectroscopy [11], optical absorption spectroscopy [12], and empirical prediction based on florescence data [34].

\begin{tabular}{lcccc}
\hline \hline Semiconducting & $E_{11}(\mathrm{eV})$ & $E_{22}(\mathrm{eV})$ & $E_{33}(\mathrm{eV})$ & $E_{44}(\mathrm{eV})$ \\
\hline$(15,10)$ EELS & 0.79 & 1.22 & 2.11 & 2.47 \\
$(15,10)$ & 0.624 (Ref. [34]) & $1.072($ Ref. [34]) & $2.13,2.15$ (Refs. [9,10]) & $2.41,2.44($ Refs. [9,10]) \\
$(15,1)$ EELS & 0.97 & 1.48 & 2.76 & 3.19 \\
$(15,1)$ (Refs. [11,12,34]) & $0.870,0.848$ & $1.337,1.379$ & $\mathrm{n} / \mathrm{a}$ & $\mathrm{n} / \mathrm{a}$ \\
& 0.869 & 1.347 & & \\
\hline \hline
\end{tabular}


TABLE III. Measured $\pi \rightarrow \pi^{*}$ "VHS peak" values for the $(13,7)$ tube, compared to values interpolated from experimental optical data [9] and optical absorption spectroscopy [12].

\begin{tabular}{lcc}
\hline \hline Metallic & $M_{11}^{ \pm}(\mathrm{eV})$ & $M_{22}^{ \pm}(\mathrm{eV})$ \\
\hline$(13,7)$ EELS & 2.15 & $3.30(-) 3.61(+)$ \\
$(13,7)$ (Refs. $[9,12])$ & $1.81(-) 1.96(+)$, & $\mathrm{n} / \mathrm{a}$ \\
& $1.857(-) 1.984(+)$ & \\
\hline \hline
\end{tabular}

three tubes there are no apparent $<5 \mathrm{eV}$ interband transition peaks in the $(15,10)$ and $(13,7)$ spectra that unambiguously could be assigned to topologically induced electron structure modification [43-45]. Such peaks are likely to arise due to excitations between defect states close to the Fermi level [43], the intensity of which should increase with increasing proximity of the electron beam to the defects in question. The present results are in agreement with the results of Senga et al. [19] who did not observe any additional contribution due to tube defects in this energy range. In terms of disordered carbonaceous material contribution, Fig. 4 shows there is a small increase in the shoulder of the $\pi+\sigma$ peak of the $(13,7)$ tube spectrum at $\geqslant \sim 20 \mathrm{eV}$ as compared to the $(15,1)$ and $(15,10)$ tube spectra. This can be attributed to a minor contribution of the $22-23 \mathrm{eV} \pi+\sigma$ plasmon of disordered carbon [42], rather than to any dependence on chirality or on the presence of topological defects. Overall, the spectra in Fig. 3 therefore show no distinct features characteristic of tube modification in proximity to the electron probe for energy losses $<\sim 20 \mathrm{ev}$.

In the recent work of Senga et al. [19], spectra acquired from metallic tubes exhibited a peak at $\sim 1 \mathrm{eV}$ that the authors attributed to the SWCNT free charge carrier plasmon, the origin of which is the collective excitation of free charge carriers in metallic or doped semiconducting SWCNTs, that propagate along the nanotube axis. The charge carrier plasmon energy is affected by finite CNT diameter [46], length, and charge carrier density [47,48]; however, the exact origin of the CNT (and graphene) charge carrier plasmon is reportedly a subject of some debate in the scientific community [48]. The charge carrier plasmon, attributed to collective excitation of the free charge carriers near the Fermi level, is thus distinctly different from the $\pi$ and $\pi+\sigma$ plasmons, which are attributed to the collective excitation of the $\pi$ and a combination of $\pi$ and $\sigma$ valence electrons, respectively. Intriguingly, no charge carrier plasmon peak was observed for the $(13,7)$ tube either in the present work or for the metallic tubes investigated by Sato and Terauchi [18]. In the present work, distinct spectral features are clearly resolved down to 0.97 and $0.79 \mathrm{eV}$ for the $(15,1)$ and $(15,10)$ tubes in Fig. 3, respectively, indicating that the experimental conditions would have allowed for the detection of such a feature at $\sim 1 \mathrm{eV}$, if present. Moreover, the absence of the charge carrier plasmon peak in the present case cannot be explained solely by differences in momentum resolution, which would have a determining effect on its visibility, as the spectra in Fig. 3 were acquired at a momentum resolution that is comparable to that used by Senga et al. [19]. The absence of an observable charge carrier plasmon peak for the $(13,7)$ tube might thus be explained either by

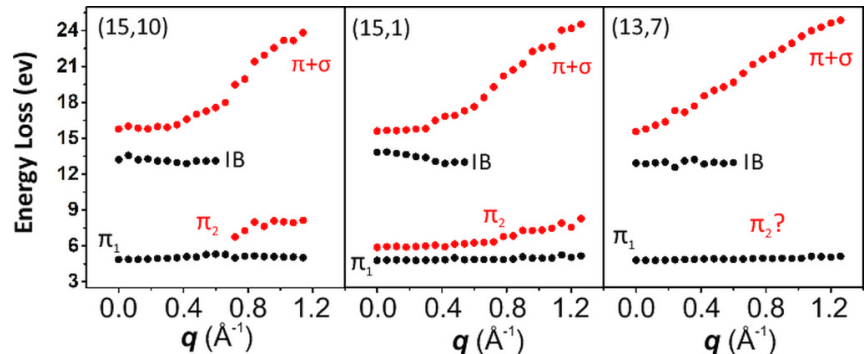

FIG. 7. SWCNT valence loss peak dispersions. Errors in energy loss peak positions are estimated to range from $< \pm 0.02 \mathrm{eV}$ at low $\boldsymbol{q}$ to a maximum of $\sim \pm 0.2 \mathrm{eV}$ for the highest $\boldsymbol{q}$ measurements. The momentum resolution is estimated to $\Delta q \approx \pm 0.25 \AA^{-1}$. The direction of $\boldsymbol{q}$ with respect to the CNT axial direction for each tube is indicated in the diffraction patterns in Fig. 1.

the $(13,7)$ charge carrier plasmon peak having a much lower relative intensity than the peaks identified by Senga et al. [19] (due to an undetermined dampening mechanism specific to the specific tube observed here) or by the energy of the peak appearing at an energy loss below the resolution of the experiment for the $(13,7)$ tube. The latter seems the most likely explanation: the charge carrier plasmon was reported in the literature to shift from $0.9 \mathrm{eV}$ to $0.6 \mathrm{eV}$ in a single defective $(12,3)$ SWCNT, when the electron beam is moved $\approx 4 \mathrm{~nm}$ from a defect-free region to a region where topological defects are present [19]. In the present case the electron beam is in relatively close proximity to a section with a higher concentration of topological defects (minimum distance of $3.2 \mathrm{~nm}$; see Fig. 1), which might explain a possible peak value of $<0.79 \mathrm{eV}$. Moreover, Senga et al. [19] suggested that unintentional doping by the TEM support grid might explain the relatively high charge carrier plasmon peaks peak observed for the metallic tubes they investigated. If in the present case the degree of a possible "unintentional doping" is significantly smaller, this could also explain why the $(13,7)$ tube charge carrier plasmon could appear at an EEL $<0.79 \mathrm{eV}$. Clearly detailed studies using monochromated STEM-EELS might significantly improve the understanding of how the charge carrier plasmon of individual SWCNTs might be affected by tube defects and doping, as well as nanotube length $[47,48]$ and diameter [46]. While a detailed study of charge carrier plasmons in individual carbon nanotubes would likely be of significant interest to the scientific community, this is beyond the scope of the present work.

\section{Momentum-resolved valence loss spectra}

The momentum dependence of the observed SWCNT loss modes can be seen in Figs. 5 and 6, with fitted peak values shown in Fig. 7. The direction of $\boldsymbol{q}$ with respect to the CNT axial direction for each tube is indicated in the diffraction patterns in Fig. 1. Spectra from all three tubes show a dispersive $\pi+\sigma$ peak (i.e., its energy changes with momentum) that broadens with increasing $\boldsymbol{q}$ and a nondispersive IB peak that becomes indistinguishable from noise for $\boldsymbol{q} \geqslant 0.6-0.7 \AA^{-1}$. As the momentum-resolved spectra were acquired in parallel, and because the spectrometer camera has a finite dynamic range, the spectral intensity $\boldsymbol{q}^{-2}$ dependence [39] necessarily results 
in spectral noise increasing significantly with increasing $\boldsymbol{q}$. This can clearly be seen in Figs. 5 and 6. While Figs. 5 and 6 show smoothed spectra as a guide to the eye, the loss mode identification (Figs. 5, 6) and peak fitting (Fig. 7) were carried out using the otherwise-unprocessed background-subtracted data, in order to avoid any potential artifact introduced by processing.

Qualitatively the $\pi \rightarrow \pi^{*}$ peaks resolved in Fig. 5 appear to be nondispersive for the $(15,10)$ and $(15,1)$ tubes, in agreement with the literature $[5,49]$. However, significant spectral noise made the unambiguous determination of their dispersion from the spectra in Fig. 5 problematic. For the $(13,7)$ tube, a lack of observable $\pi \rightarrow \pi^{*}$ peaks for the momentum-resolved spectra is attributed to a combination of spectral noise and obscuring by the ZLP tail having increased as compared to the spectra in Fig. 3 due to a relative decrease in effective energy resolution (see Sec. II).

The $(15,10)$ and $(15,1)$ tubes demonstrate arguably the most intriguing feature of these momentum-resolved spectra with a clear splitting of the $\pi$ plasmon peak into a nondispersive $\pi_{1}$ and a dispersive $\pi_{2}$ mode. While the $\pi$ peak split is identifiable in Fig. 5, this is perhaps easier to observe unambiguously in Fig. 6, which shows spectra for selected momentum transfers over a limited energy loss range. The $\pi_{1}$ mode is present in all three tubes, but the degree to which the $\pi_{2}$ mode was detected varied greatly: $\pi_{2}$ was detected for all $\boldsymbol{q}$ for the $(15,1)$ tube, only for $\boldsymbol{q} \geqslant 0.72 \AA^{-1}$ for the $(15,10)$ tube, and not at all for the $(13,7)$ tube.

Using a purpose-built stand-alone EEL spectrometer apparatus [50], a $\pi$ peak splitting has been identified in measurements averaged over "bulk samples" containing a large number of bundles of aligned SWCNTs [6-8]. Kramberger et al. [6,7] and Liu et al. [8] reported interpretations of the SWCNT $\pi$ plasmon EEL peak splitting in terms of polarization-dependent plasmon confinement. This suggests that the nondispersive $\pi_{1}$ mode corresponds to $\pi$ plasmon confinement perpendicular to SWCNT axis while the prominent dispersion of the $\pi_{2}$ mode indicates significant plasmon propagation along the SWCNT axis. While the $\pi_{1}$ mode was not identified by Pichler et al. [5] and Knupfer et al. [49], they too attributed the significant dispersion of the $\pi_{2}$ mode to polarization-dependent plasmon propagation along the CNT axes. This interpretation can be illustrated conceptually by considering a plasmon as the quasiparticle corresponding to envelopes over the collective valence electron oscillations set up by the impinging electron beam [32]. From the definition of the group velocity, the dispersion of the plasmon indicates the degree to which it is allowed to propagate in the system: a nondispersive plasmon indicates zero group velocity (i.e., a standing wave) and thus a localized mode, while a dispersive mode indicates a nonzero group velocity and thus significant plasmon propagation [27]. For detailed analysis of SWCNT $\pi$ and $\pi+\sigma$ plasmon dispersions, local field effects (LFEs) need to be considered. Kramberger et al. [6] showed by means of ab initio modeling and momentum-resolved EEL spectra that LFEs are of major importance for the $\pi$ plasmon dispersion of SWCNTs (as well as for mono- and bilayer graphene). The contribution of LFEs was attributed to the observed and predicted SWCNT $\pi$ plasmon dispersions deviating significantly from that of graphite and bundled SWCNTs.
In order to accurately interpret the observed differences in $\pi_{2}$ plasmon dispersion between the three tubes (Figs. 5-7), the experimental setup and direction of momentum selectivity must first be taken into consideration. The reported [8] and inferred $[5-7,49] \pi$ plasmon polarization dependence means the $\pi_{1}$ mode will appear at the highest relative intensity for spectra formed by collecting electrons that have imparted momentum to the sample valence $\pi$ electrons perpendicular to the nanotube axis. Conversely, the $\pi_{2}$ mode will appear at the highest relative intensity for spectra formed by collecting electrons that have imparted momentum to the sample valence $\pi$ electrons parallel to the nanotube axis. The relative orientation of the momentum-selecting slit to the tubes' axial directions is indicated schematically in the diffraction patterns in Fig. 1, which gives the direction of $\boldsymbol{q}$ for each measured tube (indicated in Fig. 1). This schematic demonstrates that if the $\pi_{2}$ mode response of all three tubes were identical, experimental geometry alone would dictate that the relative intensity of the $\pi_{2}$ mode would increase from the $(15,1)$ tube to the $(15,10)$ tube and be at its highest relative intensity for the $(13,7)$ tube. However, the spectra in Figs. 5 and 6 show clearly that this is not the case. Hence the results shown in Figs. 57 cannot be explained in terms of experimental geometry alone.

The observed differences in $\pi_{2}$ plasmon dispersion might rather be understood in terms of variable topologically induced plasmon confinement along the nanotube axis. Specifically, it is postulated that the degree to which the $\pi_{2}$ mode is confined (along the tube axis) depends on the concentration of topological defects (i.e., nonhexagonal rings) in a SWCNT. From comparing the MAADF images in Fig. 1 and the dispersions in Fig. 7 , it can be deduced that the $\pi_{2}$ mode is significantly confined at all probed wavelengths (i.e., all values of $\boldsymbol{q}$ ) in the tube containing the largest number of topological defects $(13,7)$; confined only at longer wavelengths $\left(\boldsymbol{q}<\approx 0.7 \AA^{-1}\right.$ ) for the tube containing an intermediate number of defects $(15,10)$; and allowed to propagate in the range of all measured wavelengths for the least defective tube $(15,1)$. This is consistent with a negligible effect of tube defects on $\pi_{2}$ plasmon propagation in the $(15,1)$ tube. A topologically induced $\pi_{2}$ mode confinement can be viewed as similar to the confinement of the $\pi_{1}$ mode perpendicular to the tube axis (induced by the finite diameter of the nanotube itself). The above interpretation is in good agreement with the reported identification of topologically induced $\pi$ plasmon confinement at the tip of a multilayered graphene cone [27]. One might argue that intrinsic structural periodicity along the CNT axis might also impose collective mode confinement in the system. The lattice parameters in the axial direction are (13,7): $25 \AA$, $(15,1)$ : $66 \AA$, and $(15,10)$ : $19 \AA$, which gives first Brillouin zone boundaries $(13,7): 0.13 \AA^{-1},(15,1)$ : $0.05 \AA^{-1}$, and (15,10): $0.17 \AA^{-1}$. From comparing these values to the dispersions in Fig. 7 it is clear that there is no obvious relationship between intrinsic structural periodicity along the CNT axis and the observed degree of $\pi_{2}$ plasmon confinement. Thus in the present case, $\pi_{2}$ mode confinement is attributed solely to the concentration of topological defects incorporated in the CNT walls. Conceptually, this might be understood as nonhexagonal rings in the CNT walls disrupting 
the delocalized $\pi$ states in the tube walls, which in turn disrupts $\pi$ plasmon propagation along the tube axis.

Note that for the $(15,1)$ tube, the apparent difference between the $\pi_{1}$ and $\pi_{2}$ peak energies in the " $\boldsymbol{q} \rightarrow 0$ spectrum" (Figs. 5-7) can be explained by experimental geometry, rather than being interpreted as a result of confinement. Due to a very modest momentum resolution $\left( \pm 0.25 \AA^{-1}\right)$, all momentumresolved spectra in the present work include significant contributions from a range of momentum transfers. Hence the $(15,1)$ tube " $\boldsymbol{q} \rightarrow 0$ spectrum" will include contributions from the dispersive $\pi_{2}$ mode for $\boldsymbol{q}$ up to $\approx 0.25 \AA^{-1}$. This effectively blueshifts the $\pi_{2}$ plasmon peak by an amount dependent on momentum resolution (i.e., beam convergence and effective size of spectrometer collection aperture). As the $\pi_{1}$ mode is nondispersive, no comparable shift is expected for the $\pi_{1}$ peak. Thus experimental geometry in combination with differences between $\pi_{1}$ and $\pi_{2}$ mode dispersions can be used to rationalize the measured energy difference between the $\pi_{1}$ and $\pi_{2}$ peaks in the " $\boldsymbol{q} \rightarrow 0$ spectrum" of the $(15,1)$ tube (Figs. 5-7).

In contrast, no significant differences were found between the tubes in terms of $\pi+\sigma$ or IB peak dispersions (see Figs. 5-7). Kramberger et al. [6,7] reported a $\pi+\sigma$ peak split into a dispersive and nondispersive mode for "bulk" samples of aligned tubes. As for the $\pi$ plasmon, they attributed the nondispersive nature of the $\pi+\sigma$ mode to confinement perpendicular to the nanotube axis. While due to spectral noise, a $\pi+\sigma$ peak split could not be unambiguously identified over the whole range of $\boldsymbol{q}$ values, the asymmetry of the $\pi+\sigma$ peak of the $(15,10)$ and $(15,1)$ spectra at high $\boldsymbol{q}$ in Fig. 5 appears very consistent with the result of Kramberger et al. [6,7]. In light of the above discussion on the $\pi_{2}$ mode confinement, the $\pi+\sigma$ peak might then be expected to be show nondispersive behavior for the $(13,7)$ tube. Indeed, such an effect is observed for $\mathrm{C}_{60}$ [51] as well as at the tip of a multilayer graphene cone [27]. However, no localization of the $\pi+\sigma$ mode along the tube axis is apparent for the $(13,7)$ SWCNT in Figs. 5 and 7. Thus it must be concluded that topologically induced confinement primarily affects the $\pi$ plasmon in the SWCNTs investigated in the present work. CNT $\sigma$ states are significantly more localized than $\pi$ states, which might explain the apparent difference in $\pi+\sigma$ and $\pi$ plasmon response to the presence of nonhexagonal rings incorporated in the CNT wall, at least in part. In-depth analysis of the measured $\pi$ and $\pi+\sigma$ plasmon dispersions might shed further light on the relative contributions of topological defects and chirality-dependent band structure. Due to the significant contribution of LFEs in the investigated systems [6], such a study would likely require comprehensive $a b$ initio modeling. It might also be of significant interest to investigate in future work the degree to which topological defects affect the propagation of the free charge carrier plasmon along the tube axis.

From the above discussion it is clear that topological defects significantly affect the plasmonic performance of SWCNTs. By extension, topological defects might thus affect the plasmonic response of graphene in a similar manner. As both CNTs and graphene are currently of significant interest for the development of plasmonic and optoelectronic devices [46], the presence of topological defects might therefore be important to take into consideration in future developments in this field. Furthermore, it is suggested that by accurate and deliberate introduction of topological defects, the propagation of the $\pi$ plasmon could be tailored in such a fashion as to form plasmonic conduits or "wires" and even more complex functional geometries.

\section{Spatially resolved valence loss spectra}

Returning to a more conventional STEM-EELS approach, the spatially resolved valence loss spectra of $(15,10),(15,1)$, and $(13,7)$ tubes are shown in Fig. 8 as a function of impact parameter $(\boldsymbol{b})$. The experimental procedure is indicated in the MAADF image of the $(15,1)$ tube in Fig. 1 ; the electron beam is moved progressively from the center of the tube $(\boldsymbol{b}=0 \mathrm{~nm})$ indicated by the white disks (Fig. 1.), past the tube wall $(\boldsymbol{b}=$ $0.5-1 \mathrm{~nm})$, and into vacuum $(\boldsymbol{b}>\sim 1 \mathrm{~nm})$ with increasing distance to the tube center (i.e., increasing $\boldsymbol{b}$ ). Overall, loss peak intensities decrease with increasing impact parameter for all three tubes, in agreement with the literature $[38,52,53]$. In a classical particle description, the maximum impact parameter allowed (in the adiabatic limit) can be expressed as $b_{\max }=$ $v_{0} /(E / \hbar)$, where $E$ is the energy loss and $v_{0}$ is the (relativistic) velocity of the impinging electron [32]. The possibility to excite a mode at a given $E$ for an impact parameter up to $b_{\max }$ is often referred to as inelastic delocalization. Effectively, a loss peak at a certain $E$ will decrease more in intensity with increasing $\boldsymbol{b}$ than a lower energy peak [32], as is the trend for all three tube spectra in Fig. 8.

For the $(13,7)$ tube, $\mathrm{a} \approx 5.5 \mathrm{eV}$ peak on the high-energy shoulder of the $\pi$ plasmon peak appears to increase from the tube center $(\boldsymbol{b}=0 \mathrm{~nm})$ to the tube wall $(\boldsymbol{b}=0.5-1.0 \mathrm{~nm})$. For $\boldsymbol{b}>\sim 1 \mathrm{~nm}$, qualitatively the $\approx 5.5 \mathrm{eV}$ peak decreases in intensity along with the rest of the peaks in the spectrum in agreement with inelastic scattering theory, discussed above. To understand the relative increase in $\approx 5.5 \mathrm{eV}$ peak intensity from $\boldsymbol{b}=0 \mathrm{~nm}$ to $\boldsymbol{b}=0.5-1.0 \mathrm{~nm}$, both experimental geometry and $\pi_{2}$ mode dispersion need to be taken into consideration. For the $(15,1)$ and $(15,10)$ tubes the higher energy shoulder of their respective $\pi$ peaks can be attributed to the $\pi_{2}$ mode. As discussed above, due to the use of finite beam convergence and spectrometer collection angles (see Sec. II), higher $\boldsymbol{q}$ components of the $\pi_{2}$ mode contribute to the recorded spectrum, resulting in an apparent blueshift of the $\pi_{2}$ mode, with respect to the " $\pi$ peak." As the $\pi_{2}$ mode can be attributed to the tangential tube response [38], the observed gradual decrease of the $(15,1)$ and $(15,10)$ " $\pi_{2}$ shoulder" intensities with increasing $\boldsymbol{b}$ is as expected from inelastic scattering theory.

However, as no dispersive $\pi_{2}$ mode was observed for the $(13,7)$ tube (see Figs. 5-7), the presence of the $\approx 5.5 \mathrm{eV}$ peak must be understood in terms of experimental geometry. Due to a finite beam convergence and spectrometer collection aperture (see Sec. II) the EEL spectrum is dominated by electrons having undergone momentum transfer perpendicular to the incident beam direction. Thus when the electron beam is incident on the nanotube axis $(\boldsymbol{b}=0 \mathrm{~nm})$ the spectrum is dominated by the CNT tangential response. However, when the beam is moved to the wall of the nanotube $(\boldsymbol{b}=0.5-1.0 \mathrm{~nm})$, the relative radial contribution increases significantly. As the 

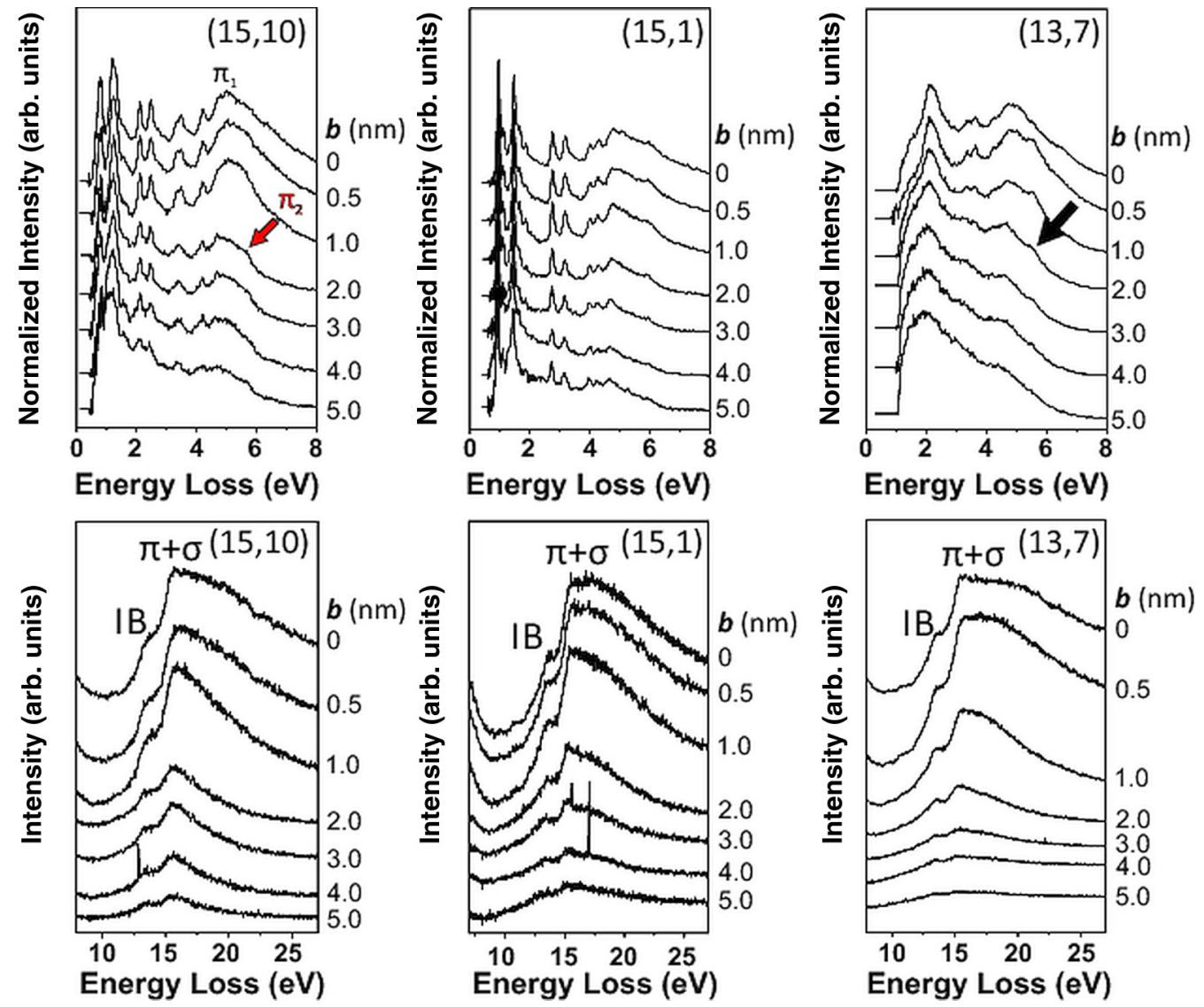

FIG. 8. Spatially resolved valence loss spectra as a function of impact parameter. The black arrow indicates a chirality-dependent radial interband transition.

$\approx 5.5 \mathrm{eV}$ peak increases significantly in intensity from $\boldsymbol{b}=0$ $\mathrm{nm}$ to $\boldsymbol{b}=0.5-1.0 \mathrm{~nm}$ for the $(13,7)$ tube, the peak is tentatively attributed to a chirality-dependent interband transition associated with the radial response of the $(13,7)$ SWCNT. Being attributed to the radial rather than the tangential response clearly differentiates the $(13,7) \approx 5.5 \mathrm{eV}$ peak from the " $\pi_{2}$ mode shoulders" of the $(15,1)$ and $(15,10)$ tubes in Fig. 8. A more accurate mode assignment of the $(13,7) \approx 5.5 \mathrm{eV}$ peak might result from theoretical modelling, possibly requiring the inclusion of depolarization and excitonic effects. This is however beyond the scope of the present work.

In light of the above discussion, a significant reduction in the high-energy shoulder of the $\pi+\sigma$ peak with increasing $\boldsymbol{b}$ for all three tubes might also be understood in terms of experimental geometry and loss mode dispersion. For $\boldsymbol{b}=0$ $\mathrm{nm}$ the $\pi+\sigma$ peak results from the spectrometer accepting electrons that have undergone a range of momentum transfers, beyond the first Brillouin zone. As the $\pi+\sigma$ mode is dispersive (see Fig. 8), it follows that the corresponding loss peak is effectively broadened on the high-energy loss side by the higher $\boldsymbol{q}$ contributions of the $\pi+\sigma$ response. With increasing $\boldsymbol{b}$ the relative magnitude of $\boldsymbol{q}$ of the tangential tube response contributing to the collected spectrum decreases. As the SWCNTs' $\pi+\sigma$ mode is tangential [38], the contribution of the high- $\boldsymbol{q}$ components of the $\pi+\sigma$ mode to the recorded loss peak decreases with increasing $\boldsymbol{b}$. Thus, the broadening of the $\pi+\sigma$ peak is reduced with increasing $\boldsymbol{b}$ for SWCNTs.
This result clearly shows that a coherent interpretation of the spatially resolved spectra results in Fig. 8 not only requires knowledge of sample orientation and experimental parameters but also of the momentum-dependence of the SWCNT loss modes themselves. In extension, similar considerations might prove useful when using valence EELS to study other lowdimensional and anisotropic samples.

\section{CONCLUSION}

STEM-EELS allows for a flexible and comprehensive characterization of the electronic structure of individual SWCNTs. Information that can be obtained includes chiral indices and structure, identification of topological defects and disordered carbonaceous material coverage (and their effect on EEL spectra), chirality-dependent interband transition energies and $\mathrm{C}-K$ ionization edge fine structure, and determination of plasmon and interband transition peak dispersions. The energy resolution provided by state-of-the-art STEM monochromators allows for spectral analysis of electronic structure comparable to that of many optical methods and dedicated EEL spectrometers. But crucially, STEM-EELS allows for the investigation of individual tubes and their defects, which is information that to a large degree is obscured in results from many optical spectroscopic methods and dedicated EEL spectrometers. In order to achieve the spatial resolution necessary to identify individual SWCNTs, the momentum 
resolution in the present work is significantly poorer than that offered by stand-alone dedicated spectrometers. However, due to the highly flexible optics of the electron microscope the intrinsic trade-off between spatial and momentum resolution can be optimized for a given experiment. Thus the momentum resolution could be made to approach that of dedicated spectrometers, if required.

The present results highlight the advantages of combining information from space- and momentum-resolved measurements when evaluating the effects of nanotube defects and chirality. Moreover, a careful comparison of spaceand momentum-resolved spectra from the same nano-object emphasizes how the relative sample orientation and choice of experimental parameters along with the dispersions of relevant loss modes might significantly affect valence EEL spectra of SWCNTs. The degree of $\pi$ plasmon confinement parallel to the SWCNT axis was shown to be dependent on the local concentration of topological defects. While the exact mechanism for confinement remains unclear, this suggests that the plasmonic response of SWCNTs could be tailored by accurate control of the topological defect concentration. By extension, a similar degree of tailoring might be possible for graphene with the aim to create plasmonic conduits or "wires" and even more complex functional geometries. Thus accurate control of the plasmonic response through the use of topological defects might prove to be beneficial in the development of SWCNTs or graphene based novel plasmonic and optoelectronic devices.

\section{ACKNOWLEDGMENTS}

The authors gratefully acknowledge O. L. Krivanek, N. Dellby, and T. C. Lovejoy (Nion Company, Kirkland, Washington, USA) for useful discussions and advice in setting up the momentum-resolved measurements, and U. Bangert (University of Limerick, Ireland) for providing CNT samples. SuperSTEM is the UK Engineering and Physical Sciences Research Council (EPSRC) National Facility for Aberration Corrected STEM. T.P.H. gratefully acknowledges the EPSRC Doctoral Prize Fellowship which funded this research.
[1] S. Iijima, Nature (London) 354, 56 (1991).

[2] R. Saito, M. Fujita, G. Dresselhaus, and M. S. Dresselhaus, Phys. Rev. B 46, 1804 (1992).

[3] N. Hamada, S.-I. Sawada, and A. Oshiyama, Phys. Rev. Lett. 68, 1579 (1992).

[4] B. J. LeRoy, S. G. Lemay, J. Kong, and C. Dekker, Appl. Phys. Lett. 84, 4280 (2004).

[5] T. Pichler, M. Knupfer, M. S. Golden, J. Fink, A. Rinzler, and R. E. Smalley, Phys. Rev. Lett. 80, 4729 (1998).

[6] C. Kramberger, R. Hambach, C. Giorgetti, M. H. Rümmeli, M. Knupfer, J. Fink, B. Büchner, L. Reining, E. Einarsson, S. Maruyama, F. Sottile, K. Hannewald, V. Olevano, A. G. Marinopoulos, and T. Pichler, Phys. Rev. Lett. 100, 196803 (2008).

[7] C. Kramberger, T. Thurakitseree, S. Maruyama, and M. Knupfer, Nanotechnology 24, 405202 (2013).

[8] X. Liu, T. Pichler, M. Knupfer, M. S. Golden, J. Fink, D. A. Walters, M. J. Casavant, J. Schmidt, and R. E. Smalley, Synth. Met. 121, 1183 (2001).

[9] K. Liu, J. Deslippe, F. Xiao, R. B. Capaz, X. Hong, S. Aloni, A. Zettl, W. Wang, X. Bai, S. G. Louie, E. Wang, and F. Wang, Nat. Nanotechnol. 7, 325 (2012).

[10] M. Y. Sfeir, T. Beetz, F. Wang, L. Huang, X. M. H. Huang, M. Huang, J. Hone, S. Brien, J. A. Misewich, T. F. Heinz, L. Wu, Y. Zhu, and L. E. Brus, Science 312, 554 (2006).

[11] S. M. Bachilo, M. S. Strano, C. Kittrell, R. H. Hauge, R. E. Smalley, and R. B. Weisman, Science 298, 2361 (2002).

[12] A. Roch, L. Stepien, T. Roch, I. Dani, C. Leyens, O. Jost, and A. Leson, Synth. Met. 197, 182 (2014).

[13] J.-C. Blancon, M. Paillet, H. N. Tran, X. T. Than, S. A. Guebrou, A. Ayari, A. S. Miguel, N.-M. Phan, A.-A. Zahab, J.-L. Sauvajol, N. D. Fatti, and F. Vallée, Nat. Commun. 4, 2542 (2013).

[14] D. Christofilos, J. C. Blancon, J. Arvanitidis, A. S. Miguel, A. Ayari, N. Del Fatti, and F. Vallée, J. Phys. Chem. Lett. 3, 1176 (2012).
[15] O. L. Krivanek, J. P. Ursin, N. J. Bacon, G. J. Corbin, N. Dellby, P. Hrncirik, M. F. Murfitt, C. S. Own, and Z. S. Szilagyi, Philos. Trans. R. Soc., A 367, 3683 (2009).

[16] O. L. Krivanek, T. C. Lovejoy, N. Dellby, T. Aoki, R. W. Carpenter, P. Rez, E. Soignard, J. Zhu, P. E. Batson, M. J. Lagos, R. F. Egerton, and P. A. Crozier, Nature (London) 514, 209 (2014).

[17] M. Mukai, J. S. Kim, K. Omoto, H. Sawada, A. Kimura, A. Ikeda, J. Zhou, T. Kaneyama, N. P. Young, J. H. Warner, P. D. Nellist, and A. I. Kirkland, Ultramicroscopy 140, 37 (2014).

[18] Y. Sato and M. Terauchi, Microsc. Microanal. 20, 807 (2014).

[19] R. Senga, T. Pichler, and K. Suenaga, Nano Lett. 16, 3661 (2016).

[20] H. Zhu, K. Suenaga, A. Hashimoto, K. Urita, and S. Iijima, Chem. Phys. Lett. 412, 116 (2005).

[21] M. Gao, J. M. Zuo, R. D. Twesten, I. Petrov, L. A. Nagahara, and R. Zhang, Appl. Phys. Lett. 82, 2703 (2003).

[22] L.-C. Qin, Phys. Chem. Chem. Phys. 9, 31 (2007).

[23] R. Saito, G. Dresselhaus, and M. S. Dresselhaus, Phys. Rev. B 61, 2981 (2000).

[24] M. K. Kinyanjui, C. Kramberger, T. Pichler, J. C. Meyer, P. Wachsmuth, G. Benner, and U. Kaiser, Europhys. Lett. 97, 57005 (2012).

[25] P. Wachsmuth, R. Hambach, M. K. Kinyanjui, M. Guzzo, G. Benner, and U. Kaiser, Phys. Rev. B 88, 075433 (2013).

[26] S. C. Liou, C. S. Shie, C. H. Chen, R. Breitwieser, W. W. Pai, G. Y. Guo, and M. W. Chu, Phys. Rev. B 91, 045418 (2015).

[27] F. S. Hage, Q. M. Ramasse, D. M. Kepaptsoglou, Ø. Prytz, A. E. Gunnaes, G. Helgesen, and R. Brydson, Phys. Rev. B 88, 155408 (2013).

[28] E. Najafi, A. P. Hitchcock, D. Rossouw, and G. A. Botton, Ultramicroscopy 113, 158 (2012).

[29] U. Falke, A.-K. Weber, and J. Ullmann, Microsc. Microanal. Microstruct. 6, 113 (1995).

[30] R. Saito, M. Fujita, G. Dresselhaus, and M. S. Dresselhaus, Appl. Phys. Lett. 60, 2204 (1992). 
[31] D. Rossouw, G. A. Botton, E. Najafi, V. Lee, and A. P. Hitchcock, ACS Nano 6, 10965 (2012).

[32] R. F. Egerton, Electron Energy-Loss Spectroscopy in the Electron Microscope (Springer, New York, 2011).

[33] K. Suenaga and M. Koshino, Nature (London) 468, 1088 (2010).

[34] R. B. Weisman and S. M. Bachilo, Nano Lett. 3, 1235 (2003).

[35] A. G. Marinopoulos, L. Reining, A. Rubio, and V. Olevano, Phys. Rev. B 69, 245419 (2004).

[36] D. Novko, V. Despoja, and M. Šunjić, Phys. Rev. B 91, 195407 (2015).

[37] E. Malić, M. Hirtschulz, F. Milde, Y. Wu, J. Maultzsch, T. F. Heinz, A. Knorr, and S. Reich, Phys. Rev. B 77, 045432 (2008).

[38] O. Stéphan, D. Taverna, M. Kociak, K. Suenaga, L. Henrard, and C. Colliex, Phys. Rev. B 66, 155422 (2002).

[39] H. Raether, Excitations of Plasmons and Interband Transitions by Electrons (Springer-Verlag, New York, 1980).

[40] A. G. Marinopoulos, L. Reining, and A. Rubio, Phys. Rev. B 78, 235428 (2008).

[41] F. Bassani and G. Pastori Parravicini, Il Nuovo Cimento B (19651970) 50, 95 (1967).

[42] H. Daniels, R. Brydson, B. Rand, and A. Brown, Philos. Mag. 87, 4073 (2007).
[43] F. S. Hage, D. M. Kepaptsoglou, C. R. Seabourne, Q. M. Ramasse, A. J. Scott, O. Prytz, A. E. Gunnaes, and G. Helgesen, Nanoscale 6, 1833 (2014).

[44] T. Stockli, J.-M. Bonard, A. Chatelain, Z. L. Wang, and P. Stadelmann, Appl. Phys. Lett. 80, 2982 (2002).

[45] W. Zhou, J. Lee, J. Nanda, S. T. Pantelides, S. J. Pennycook, and J.-C. Idrobo, Nat. Nanotechnol. 7, 161 (2012).

[46] F. J. García de Abajo, ACS Photonics 1, 135 (2014).

[47] Q. Zhang, E. H. Hároz, Z. Jin, L. Ren, X. Wang, R. S. Arvidson, A. Lüttge, and J. Kono, Nano Lett. 13, 5991 (2013).

[48] T. Morimoto, S.-K. Joung, T. Saito, D. N. Futaba, K. Hata, and T. Okazaki, ACS Nano 8, 9897 (2014).

[49] M. Knupfer, T. Pichler, M. S. Golden, J. Fink, A. Rinzler, and R. E. Smalley, Carbon 37, 733 (1999).

[50] J. Fink, in Advances in Electronics and Electron Physics (Academic Press, London, 1989), p. 121.

[51] T. Pichler, M. Knupfer, M. S. Golden, J. Fink, and T. Cabioc'h, Phys. Rev. B 63, 155415 (2001).

[52] B. W. Reed and M. Sarikaya, Phys. Rev. B 64, 195404 (2001).

[53] M. Kociak, L. Henrard, O. Stéphan, K. Suenaga, and C. Colliex, Phys. Rev. B 61, 13936 (2000). 